\title{
Research on Optimal Acceleration and Deceleration Control Algorithm for High-speed Parallel Manipulator
}

\author{
Lingzhen Sun ${ }^{1}$, Shuo $\mathrm{Ye}^{2, *}$ \\ ${ }^{1}$ Guangzhou Huali Science And Technology Vocational College, Guangzhou 511325, China \\ ${ }^{2}$ Huali College Guangdong University of Technology, Guangzhou 511325, China \\ *Corresponding Author email: 61358206@qq.com
}

Keywords: High speed parallel manipulator; trajectory planning; simplified rigid body dynamics model

\begin{abstract}
Supported by the National Natural Science Foundation of China (NSFC) "Integrated optimization design of high-speed parallel mechanism based on motor performance parameters". Taking Delta parallel manipulator with three degrees of freedom as an example, the rigid body dynamics modeling of manipulator is taken as an example. And motion control methods from two angles. The control method of improving manipulator speed and grasping and releasing precision based on the dynamic model of manipulator is studied systematically. With the help of vector method, constraint equation and kinetic energy theorem, the position inverse solution model, velocity Jacobian matrix and mass inertia matrix of manipulator are constructed respectively. On this basis, the rigid body dynamic model is established by using the principle of virtual work, which provides the necessary theoretical premise and technical basis for trajectory planning and manipulator control. The algorithm can effectively compensate the tracking error caused by dynamic factors by combining PID feedback with nonlinear feedback. In addition, the influence of various trajectory planning methods on control is compared, and the optimal control is selected to improve the control performance.
\end{abstract}

\section{Introduction}

With the continuous development and progress of China's manufacturing industry, the application of various types of industrial robots in the manufacturing industry is increasing [1]. Especially in the electronic, pharmaceutical, food and other industries in the sorting, testing and packaging operations, rapid and accurate positioning of the robot is becoming more widely used [2]. At present, the above-mentioned operation mainly depends on the serial manipulator, but the serial manipulator needs to install a driving device on each joint, so the motion inertia of each arm is large, it is difficult to achieve high-speed or ultra-high-speed operation. The study of series mechanism started earlier, and its kinematics, dynamics and trajectory planning are relatively perfect [3]. However, the actuator of the serial manipulator needs to be installed on each joint, which leads to excessive inertia of the manipulator, so the dynamic characteristics are not good, affecting the positioning accuracy. Therefore, as a knowledge-intensive machine, high-speed parallel manipulator has incomparable advantages in high-speed packaging, insertion and high-speed grasping of materials in light industry [4].

\section{Kinematics and rigid body dynamics analysis}

The kinematics model of manipulator is the basis of establishing dynamic model and trajectory planning and motion control, so it is necessary to establish the kinematics model of manipulator first. For high-speed parallel manipulators, due to the influence of centrifugal force, Coriolis force and gravity term, most of the linear single-axis controllers such as PD and PID used in commercial manipulators can not achieve satisfactory control quality. Kinematics analysis involves solving the 
mapping relations between joint variables and operation space variables, such as position, velocity and acceleration, with known mechanism scale parameters. It can be generally summed up as positive and inverse problems. Delta manipulator is mainly composed of static platform, moving platform and three branched chains connecting two platforms. Servo motor and reducer are installed on the static platform. Each branched chain is composed of active arm and driven arm. The acceleration and deceleration curves of the parallel manipulator require that the speed and acceleration of the end effector are 0 . It can reach the peak velocity in the prescribed time, and the displacement function is third-order derivative and continuous, and the fourth-order derivative is upper bound. The dynamic model of the parallel manipulator describes the mapping relationship between the position, velocity, acceleration and the torque of each driving joint. It has a direct impact on the control accuracy and efficiency of the dynamic control method.

Using the inverse solution model of position, velocity and acceleration of Delta manipulator, the angle, angular velocity and angular acceleration curves of each active arm joint in joint space during the motion can be obtained as shown in Figure 1.

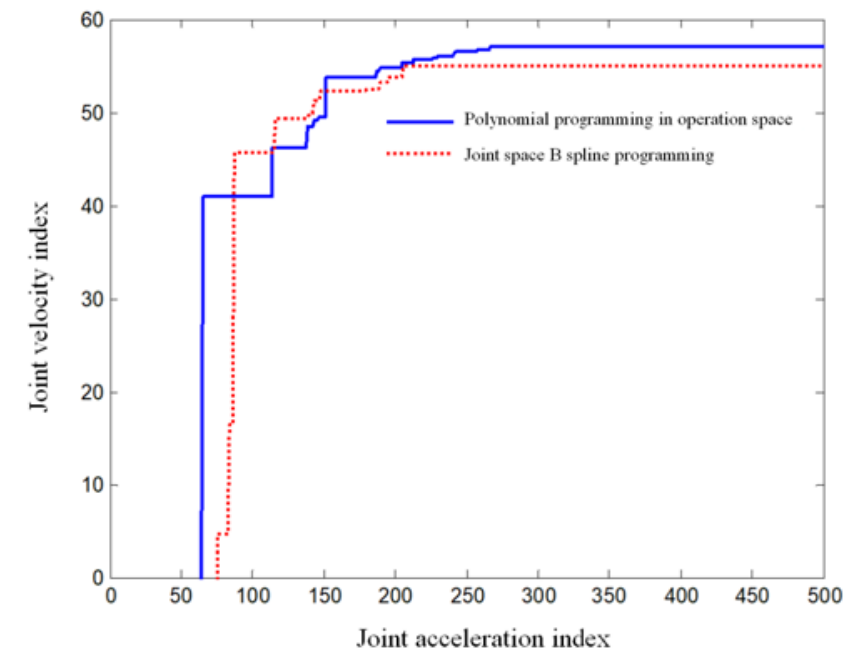

Figure 1 Comparison of joint motion parameters

The maximum joint angular velocity and angular acceleration in Figure 1 are collated and data obtained are shown in Table 1.

Table 1 Maximum joint speed and acceleration in manipulator motion

\begin{tabular}{ccccccc}
\hline $\begin{array}{c}\text { Trajectory } \\
\text { planning } \\
\text { method }\end{array}$ & Joint 1 & Joint 2 & Joint 3 & Joint 1 & Joint 2 & Joint 3 \\
\cline { 2 - 7 } & & & & & & \\
\hline $\begin{array}{c}\text { Operation } \\
\text { space } \\
\text { polynomial } \\
\text { programming } \\
\text { joint space }\end{array}$ & 8.259 & 9.567 & 9.598 & 361.1 & 142.6 & 325.5 \\
$\begin{array}{c}\text { B spline } \\
\text { programming }\end{array}$ & 8.598 & 5.561 & 10.256 & 125.9 & 111.2 & 186.3 \\
\hline
\end{tabular}

The inverse kinematics model is mainly used to establish the mapping relationship between the space position, velocity, acceleration of the terminal platform and the rotation angle, angular velocity and angular acceleration of the active arm. The inverse dynamics of rigid body involves the angular acceleration, torque and power converted to the active joint under the condition that the inertia parameters and the motion law of the end of the mechanism are known. It is used to lay the foundation for the design analysis and control system establishment of the organization. For parallel mechanism, 
vector method is usually used to establish kinematics model. The main purpose of forward kinematics is to find the position of the end of the manipulator by the known joint angles. It is mainly used in the motion simulation of the robot system. At the same time, it can be used for trajectory planning of joint space to obtain the end movement state of manipulator. Detailed derivation of forward kinematics model of Delta mechanism is given. In view of the need of trajectory planning and dynamic control, the final derivation is given here. Model-based computational torque control, also known as inverse dynamics method, is an example of nonlinear system feedback linearization method applied to second-order nonlinear systems. The highly nonlinear model is transformed into the corresponding linear system by the combination of PID feedback and nonlinear feedback. According to the linear system theory, each joint is synchronously closed-loop controlled, and the output torque of the servo motor is continuously adjusted to compensate the tracking error, so that it tends to the ideal value.

The derivative of absolute acceleration to time is obtained by using gradient function, and the jump curves of the end effector to time under two trajectory planning methods are obtained, as shown in Figure 2.

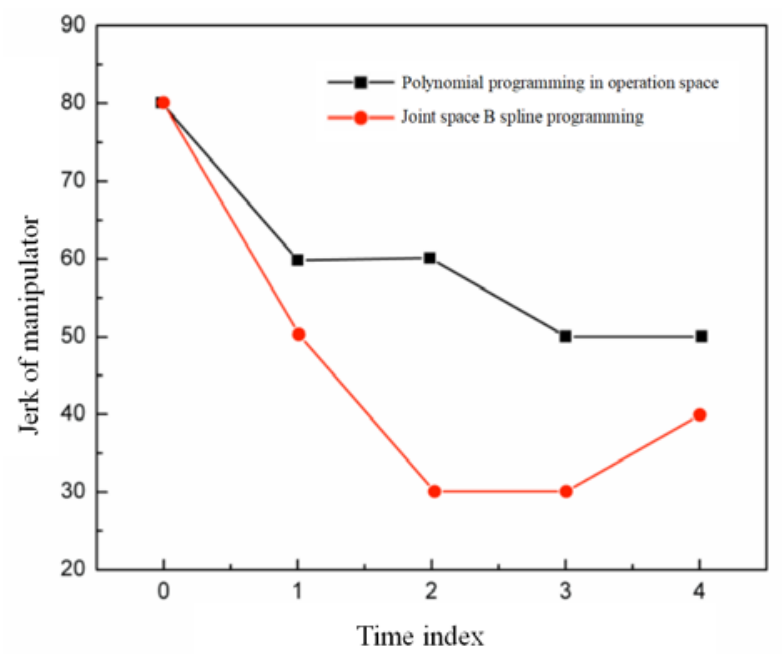

Figure 2 Terminal jump of manipulator

Table 2 shows the maximum angular velocity, maximum angular acceleration, maximum input torque and maximum power in different trajectory planning methods and transition modes.

Table 2 Control quality of computed torque control under different trajectory modes

\begin{tabular}{|c|c|c|c|c|c|}
\hline $\begin{array}{l}\text { Trajectory } \\
\text { planning } \\
\text { method }\end{array}$ & $\begin{array}{l}\text { Transitional } \\
\text { form }\end{array}$ & $\begin{array}{l}\text { Maximum } \\
\text { angular } \\
\text { velocity }\end{array}$ & $\begin{array}{l}\text { Maximum } \\
\text { angular } \\
\text { acceleration }\end{array}$ & $\begin{array}{l}\text { Maximum } \\
\text { input torque }\end{array}$ & $\begin{array}{l}\text { Maximum } \\
\text { power }\end{array}$ \\
\hline $\begin{array}{l}\text { Operation } \\
\text { space }\end{array}$ & $\begin{array}{l}\text { Straight line } \\
\text { transition }\end{array}$ & 15.152 & 351.9 & 55.21 & 651.9 \\
\hline $\begin{array}{l}\text { polynomial } \\
\text { programming } \\
\text { joint space }\end{array}$ & Arc transition & 16.451 & 321.5 & 55.65 & 595.4 \\
\hline \multirow{2}{*}{$\begin{array}{c}\text { B spline } \\
\text { programming }\end{array}$} & $\begin{array}{l}\text { Straight line } \\
\text { transition }\end{array}$ & 14.567 & 262.1 & 59.26 & 562.9 \\
\hline & Arc transition & 15.285 & 356.6 & 51.39 & 456.5 \\
\hline
\end{tabular}

\section{Dynamic control method}

In order to simulate and analyze different dynamic control methods, the model of servo control system should be established firstly. Diamond high-speed parallel mechanism is an electromechanical time-varying system. The servo motor is constantly subjected to time-varying load from different positions and poses of the mechanical parts in the working process. Therefore, adaptive control is needed in principle. For common commercial servo systems, the three-loop closed-loop PID control of position loop, speed loop and current loop is usually used. However, 
under the constraints of hardware conditions, most of the PID parameters of the popular controller in the market can not be adjusted online. Therefore, it is necessary to adjust the PID parameters offline reasonably, so as to optimize the dynamic quality of the system in the whole workspace. For motor servo system with three-loop closed-loop control, PI control is usually used in current loop and speed loop, and PID control is used in position loop. The objective function of classical PID parameter tuning is essentially such as overshoot, rising time, adjusting time and so on. In the current commonly used PID tuning methods, it can be divided into two types: identification method and rule method. The identification method is mainly applicable to the known control system models. But when the control parameters are unknown, the system identification is used to obtain the control parameters. The classical methods include pole assignment principle, zero-pole cancellation principle, amplitude-phase margin method and so on.

Figure 3 and Table 3 show the angular displacement error of the servo motor and the set trajectory and actual trajectory of the end effector in the operating space.

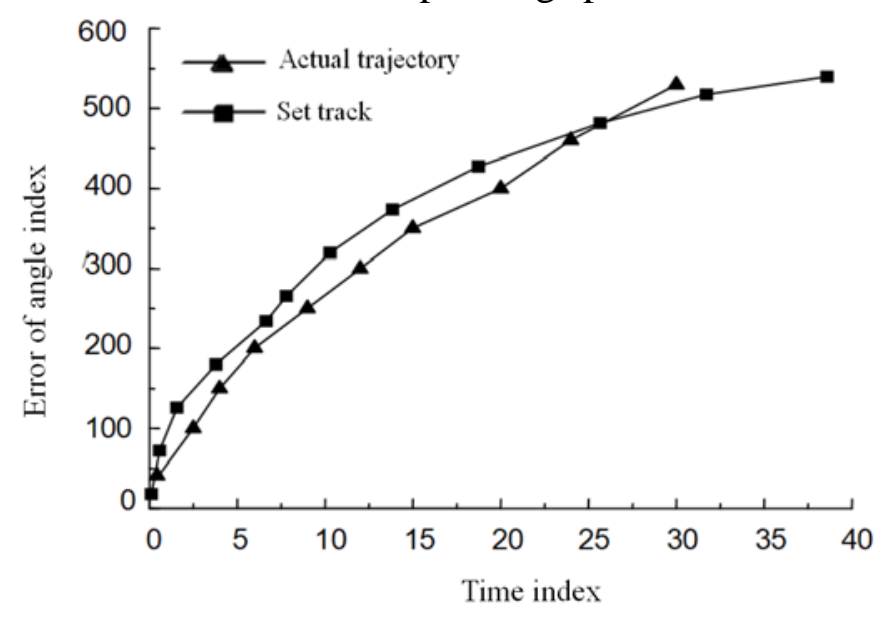

Figure 3 Angular displacement error

Table 3 Angular displacement error exponent

\begin{tabular}{ccc}
\hline & Set track & Actual trajectory \\
\hline Angle error & 315 & 350 \\
Operation time of end effector & 20 & 18 \\
\hline
\end{tabular}

Because the control system is a complex time-varying system, the effect of Z-N tuning is limited, and the overshoot of step response is easy to occur. At the same time, this method has a higher requirement for the control knowledge and setting experience of the developer, and the setting result can not be guaranteed. Therefore, this paper introduces a self-tuning method based on genetic algorithm. The self-tuning of PID parameters refers to the process of solving the controller parameters and updating the PID control according to the system parameters in the control process. Genetic algorithm (GA) is a kind of probabilistic search algorithm which simulates the genetic evolution rules of survival of the fittest and survival of the fittest in nature. It has self-adaptive ability and is a global intelligent algorithm. The genetic algorithm starts with the population with possible solution set, and establishes the mapping relationship between phenotype and genotype, that is, coding. Thus the problem is mapped from the solution space to the encoding space, and the encoding in the encoding space corresponds to the solution of the problem one by one. The encoding is called an individual or chromosome. Then, according to the principle of survival of the fittest and survival of the fittest, the initial population evolves from generation to generation, and an approximate solution is generated. In each generation, individual selection is based on the fitness of different individuals in the problem domain. At the same time, the genetic operators in genetics are used for reference to cross-combine and mutate different individuals, so as to produce a new population corresponding to a new set of solutions.

The sinusoidal position signal with frequency applied to the test joint is still observed under 
different control methods. As shown in Figure 4 and Table 4.

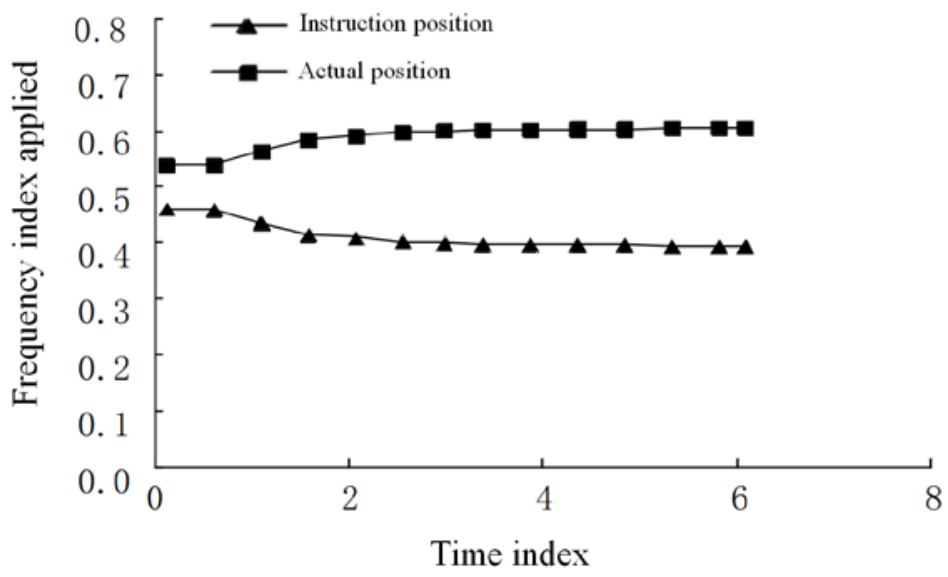

Figure 4 Sinusoidal motion of high frequency in uniaxial system

Table 4 Simulation index of high frequency sinusoidal motion for uniaxial system

\begin{tabular}{ccc}
\hline & Instruction position & Actual position \\
\hline $\begin{array}{c}\text { Frequency of joint } \\
\text { application } \\
\text { Time }\end{array}$ & 0.4 & 0.6 \\
& 3 & 2 \\
\hline
\end{tabular}

\section{Conclusion}

The complete rigid body dynamic model based on virtual work principle is not suitable for real-time torque calculation of dynamic control system because of the complexity of the calculation of the components of the driven arm. Therefore, a new rigid body dynamic model simplification method based on the equivalent mass distribution of the driven arm is proposed, which is simplified from inertial force, gravity and torque. By means of vector method, constraint equation, kinetic energy theorem and virtual work principle, the inverse position solution model, velocity Jacobian, matrix mass inertia matrix and rigid body dynamic model of manipulator are established respectively. By equivalent the spatial mass inertia model of the manipulator to the rotor inertia of the motor, the PID parameters of the closed-loop kinematics control are tuned by Z-N tuning and self-tuning method based on genetic algorithm. The tuning results of the self-tuning method based on genetic algorithm have better dynamic characteristics. It has certain practical value for the kinematics and dynamics control method based on PID control in engineering application.

\section{References}

[1] Silva M M D, Oliveira L P R D, Brüls O, et al. Integrating structural and input design of a 2-DOF high-speed parallel manipulator: A flexible model-based approach [J]. Mechanism \& Machine Theory, 2010, 45(11):1509-1519.

[2] Yuhang L I, Mei J, Liu S, et al. Dynamic Dimensional Synthesis of a 4-DOF High-speed Parallel Manipulator [J]. Journal of Mechanical Engineering, 2014, 50(19):32.

[3] Tao S, Song Y M, Kai Y. Kineto-static analysis of a novel high-speed parallel manipulator with rigid-flexible coupled links [J]. Journal of Central South University, 2011, 18(3):593.

[4] Yang Z, Feng W, Jiang W, et al. Digital platform-based multi-domain virtual prototype simulation on a high-speed parallel manipulator[J]. Robotica, 2012, 30(5):827-835. 